\title{
Oral corticosteroid prescribing habits of Canadian Otolaryngologist-Head and Neck Surgeons
}

\author{
Saad Ansari ${ }^{1}$, Brian W. Rotenberg ${ }^{1}$ and Leigh J. Sowerby ${ }^{1,2^{*}}$
}

\begin{abstract}
Background: Oral corticosteroids (OCSs) are widely prescribed in Otolaryngology-Head \& Neck surgery (OtoHNS). There is evidence in the literature regarding specific dosing regimens. However, it is not known to what extent these recommendations are being implemented in practice.

Methods: An anonymous online survey was sent to Canadian Society of Otolaryngology-Head and Neck Surgery members $(N=696)$. Dosing, frequency and tapering of OCSs were assessed in acute rhino-sinusitis (ARS), chronic rhino-sinusitis with (CRSwP) and without polyps (CRSsP), sudden sensori-neural hearing loss (SSNHL), and idiopathic facial nerve/Bell's palsy (IFN). Participants were asked to complete for conditions treated and results were compared with current guidelines. Development of prescribing habits and observed complications were also explored.
\end{abstract}

Results: 124 surveys (18 \%) were completed. In CRSwP ( $N=98)$, the median dose was 50 mg (Range: 10-100 mg) and the average duration was 8 days (Range: $1-21$ days). In CRSsP $(N=29)$, the median dose was 50 mg (Range: 20-80 $\mathrm{mg}$ ) and the average duration was 8 days (Range: 1-14 days). In SSNHL ( $N=118$ ), the median dose was $60 \mathrm{mg}$ (Range: 10-120 mg) and the average duration was 10 days (Range: $1-21$ days). In IFN ( $N=108)$, the median dose was $50 \mathrm{mg}$ (Range: 10-100 mg) and the average duration was 10 days (Range: 1-21 days). Tapering dosages were used in treating CRSwP (64 \%), CRSsP (62 \%), ARS (44 \%), SSNHL (60 \%) and IFN (53 \%). Respondents most frequently perceived "Mentor/Preceptor Guidance" as a source of their prescribing habits.

Conclusion: There is significant heterogeneity in OCS prescribing habits despite the availability of fairly consistent evidence in the literature for some of the surveyed conditions. Improvements in standardization should be made with the aim of enhancing outcomes and reducing complications.

Keywords: Oral corticosteroids, Otolaryngology, Dosing regimen, Evidence-based medicine, Chronic Rhinosinusitis, Taper, Sudden Sensori-neural Hearing Loss, Facial Nerve Palsy

\section{Background}

Oral corticosteroids (OCSs) are commonly used for a variety of different diseases. In 2013, Overman et al. determined that $1.2 \%$ of the US population over age 20 were prescribed OCSs, translating into over 2.5 million

\footnotetext{
* Correspondence: leigh.sowerby@sjhc.london.on.ca

Meeting presentation Poster presentation at the Canadian Society of Otolaryngology-Head and Neck Surgery Annual General Meeting in Winnipeg, Ontario, Canada in June 2015.

1 Department of Otolaryngology-Head \& Neck Surgery, Western University, London, ON, Canada

2Department of Otolaryngology-Head \& Neck Surgery, St. Joseph's Hospital, Room B2-501, 268 Grosvenor Street, London, ON N6A 4V2, Canada
}

people [1]. In the field of Otolaryngology-Head \& Neck Surgery (OtoHNS), OCSs are used for several indications, including chronic rhinosinusitis, sudden sensorineural hearing loss, and idiopathic facial nerve, or Bell's, palsy.

Due to the significant side effect profile, OCSs are often reserved as part of maximal medical therapy when other treatments have not been successful. Complications such as avascular necrosis of the hip, immunodeficiency, weight gain, insomnia, and psychosis have been well described in the literature [2]. While some are idiosyncratic reactions, 
most side effects have been shown to correlate with increasing doses of OCSs [3]. Therefore, the dosing of OCSs is important to provide maximal benefit while minimizing potential side effects.

A 2013 UK study by Sylvester et al. qualitatively characterized OCS prescribing habits as part of maximal medical therapy in chronic rhinosinusitis, with $66 \%$ rarely or never prescribing OCSs [4]. Of those that did, $42 \%$ utilized a duration of 0-5 days, $29 \%$ for $6-10$ days and $29 \%$ for 11-15 days - suggesting that there is significant heterogeneity in prescribing practice in the UK. Kaszuba \& Stewart showed that $36 \%$ of Otolaryngologist-Head \& Neck surgeons used OCSs in chronic rhinosinusitis [5]. This study also concluded that maximal medical management was influenced mainly by personal clinical experiences rather than evidence in the literature. Similar studies have not been conducted in Canada regarding OCS prescribing habits.

The aim of this study is to characterize OCS prescribing habits of Canadian Otolaryngologist-Head \& Neck surgeons for chronic rhinosinusitis, acute rhinosinusitis, sudden sensori-neural hearing loss, and idiopathic facial nerve (Bell's) palsy. While the evidence for dosage is heterogeneous for some conditions, such as chronic rhinosinusitis, a much clearer consensus on dosage exists for others. This study hopes to provide a glimpse into the status of evidence-based practice for prescribing OCSs in Canadian OtoHNS.

\section{Methods}

Formal ethics approval was obtained through the Western University Research Ethics Board (Board number 105523) prior to beginning the study. An anonymous nationwide survey was conducted through an online survey program (QuestionPro.com ${ }^{\circ}$ ) and electronically distributed to all active members of the Canadian Society of Otolaryngology - Head and Neck Surgery's mailing list $(n=696)$ between October and November 2014. A reminder email was sent approximately 3 months after initial distribution and participants were incentivized with a gift card draw at completion of the study. A cover letter accompanied the survey to outline issues of consent and to disclose the study's goal of characterizing prescribing habits of Canadian Otolaryngologist-Head \& Neck surgeons. Respondents were notified that no identifying data would be included in the study.

Demographic data collected focused on the nature of respondents' current practices. Indication, initial dose, duration, frequency, and use of taper were described for five common indications of OCSs in OtoHNS. These were chronic rhinosinusitis with polyposis (CRSwP), chronic rhinosinusitis without polyposis (CRSsP), acute rhinosinusitis (ARS), sudden sensori-neural hearing loss (SSNHL), and idiopathic facial nerve (Bell's) palsy (IFN). Respondents were also asked to describe influences that helped to establish personal dosing regimens as well as observed complications with the use of OCSs in practice (Fig. 1).

\section{Results}

Out of the 696 survey requests sent to members of the Canadian Society of Otolaryngology-Head and Neck Surgery, 124 surveys were returned fully completed (18\% response rate). The majority of respondents were surgeons

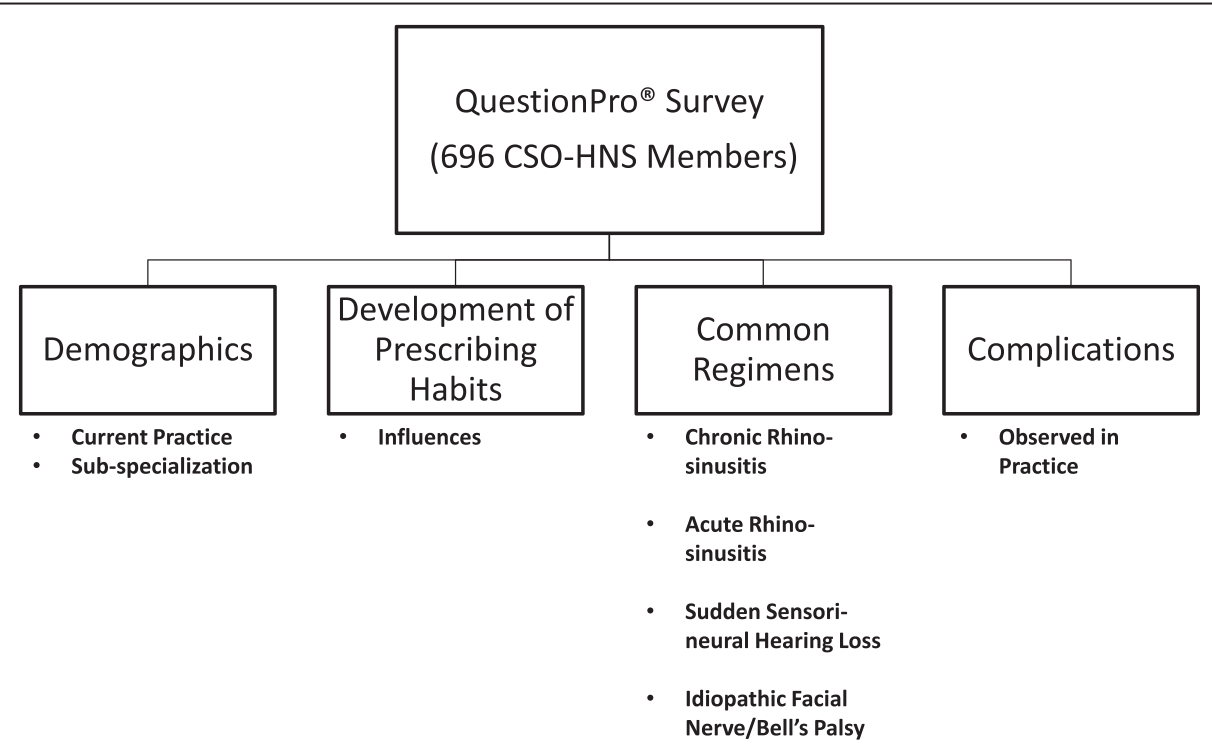

Fig. 1 Components of the online survey. Respondents were asked to answer questions regarding personal demographics, development of prescribing habits, and specific dosing regimens for CRSwP, CRSSP, ARS, SSNHL, and IFN. Respondents were also asked to describe complications observed in their practice 
in active practice $(87 \%)$. There were slightly more community practitioners (55\%) than academic practitioners (40\%). The remaining $5 \%$ had a mixed academic and community practice. The two most-represented subspecialties were General Otolaryngology (52\%) and Rhinology (27\%). These were followed by Pediatrics (23\%), Head and Neck (20\%), Otology (19\%), Facial Plastic and Reconstructive Surgery (17\%), Laryngology (12 \%) and other $(4 \%)$. The average number of OCSs prescribed by respondents was 6 prescriptions per month (range: 0-40 prescriptions).

The most common self-described OCS prescribing influence was a respondent's mentor or preceptor (78\%), followed by personal experience (64\%) and clinical guidelines (59 \%) (see Fig. 2).

\section{Chronic rhinosinusitis}

In chronic rhinosinusitis, $79 \%$ of respondents prescribed OCSs for CRSwP and $23 \%$ for CRSsP. The most common reasons in both cases were "as part of maximal medical therapy" and "symptomatic exacerbation". In CRSwP, there were 12 unique doses described by respondents. The median starting dose was $50 \mathrm{mg}$ with a range between 10 and $100 \mathrm{mg}$. The average duration was 8 days with a range between 1 and 21 days. In CRSsP, there were 7 unique doses used by respondents. The median dose was $50 \mathrm{mg}$ with a range between 20 and $80 \mathrm{mg}$. The average duration was 8 days with a range between 1 and 14 days. Tapers were used by approximately two-thirds of those that used corticosteroids for both conditions (Table 1, Figs. 3 \& 4).

\section{Acute rhinosinusitis}

In acute rhinosinusitis, $7 \%$ of respondents reported prescribing OCSs. All nine respondents had unique dosing regimens. The median dose was $50 \mathrm{mg}$ with a range between 25 and $60 \mathrm{mg}$. The average duration was 6 days with a range between 2 and 10 days. A total of $44 \%$ used a taper (Table 1, Figs. 3 \& 4).

\section{Sudden sensori-neural hearing loss}

In SSNHL, an overwhelming majority of respondents used OCSs (95\%). The most common reason to initiate therapy was to improve hearing. There were 11 unique dosing regimens described. The median starting dose was $55 \mathrm{mg}$ with a range between 10 and $100 \mathrm{mg}$. The most common starting dose was between 40 and $60 \mathrm{mg}$ of prednisone, with 98 (80\%) of respondents doing so. The average duration was 10 days with a range between 1 and 21 days. In this group of respondents, $60 \%$ utilized a taper (Table 1, Figs. 3 \& 4). Approximately $80 \%$ (98/123) of respondents used a starting dose between 40 and $60 \mathrm{mg}$.

\section{Idiopathic facial nerve (Bell's) palsy}

For IFN palsy, $87 \%$ of respondents prescribed OCSs. There were 9 unique dosing regimens described. The median dose was $50 \mathrm{mg}$ with a range between 10 and $100 \mathrm{mg}$. Only 83 (67\%) of respondents used a starting dose of prednisone between 40 and $60 \mathrm{mg}$. The average duration was 9 days with a range between 1 and 21 days. Of these respondents, $53 \%$ employed a taper (Table 1, Figs. 3 \& 4).

\section{Complications}

When asked about complications observed in practice, $30 \%$ of respondents had personally managed patients with complications from the use of OCSs (Fig. 5). Insomnia, weight gain and gastrointestinal symptoms were most commonly described. Other rare complications were also mentioned including avascular necrosis, adrenal suppression, and hypoglycaemia (Fig. 6).

\section{Discussion}

This study has characterized the corticosteroid prescribing habits of Canadian Otolaryngologist-Head \& Neck

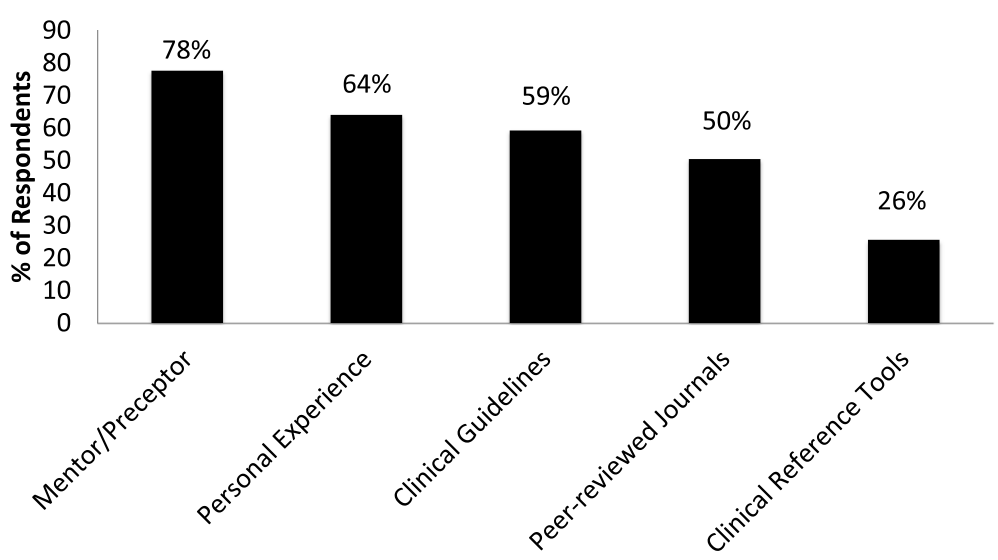

Fig. 2 Influences for current oral corticosteroid prescribing habits of survey respondents 
Table 1 Dosing regimens responses for CRS, ARS, SSNHL, and IFN/Bell's Palsy. This table shows the use of OCSs, number of unique dosing regimens reported, median starting dose, average duration, and taper utilization for CRSwP, CRSsP, ARS, SSNHL, and IFN

\begin{tabular}{llllll}
\hline Indication & Use (\% of respondents) & Unique dosing regimens & Median dose with ranges & Duration with ranges & Taper (\% of respondents) \\
\hline CRSwP & 79 & 12 & $50(10-100)$ & $8(1-21)$ & 64 \\
CRSsP & 23 & 7 & $50(20-80)$ & $8(1-14)$ & 62 \\
ARS & 7 & 9 & $50(25-60)$ & $6(2-10)$ & 44 \\
SSNHL & 95 & 11 & $55(10-120)$ & $10(1-21)$ & 60 \\
IFN/Bell's Palsy & 87 & 9 & $50(10-100)$ & $9(1-21)$ & 53 \\
\hline
\end{tabular}

surgeons for five common conditions in OtoHNS. The $18 \%$ response rate, which is similar to previous survey studies of Canadian Otolaryngologist-Head and Neck surgeons, provides a well-balanced representation of community and academic practice respondents [6-9]. The results demonstrate the significant variability in prescribing habits, as evidenced by the high number of unique dosing regimens for each condition. Even greater variation would likely have been seen with a higher response rate, as the findings of this study are in keeping with the heterogeneity seen in previous surveys for OCS usage [4]. This is likely to be expected as mentor and preceptor habits along with personal experience were most commonly selected as being influences on respondents' prescribing habits.

For CRSwP, the 2011 Canadian Society of OtolaryngologyHead and Neck Surgery (CSO-HNS) clinical practice guidelines suggest a two-week course of OCS to aid in treatment [10]. It does not elaborate on dosing regimens. A 2013 International Forum of Allergy \& Rhinology review by Poetker et al. provides a more specific regimen of $25-60 \mathrm{mg}$ for 7-14 days with Level A evidence from Level 2-4 studies [11]. The Canadian
Family Physician guidelines also provide a dosing regimen of a two-week course of prednisone with a taper [12]. An example regimen was given for $30 \mathrm{mg}$ per day for 4 days, then reduce the dose by $5 \mathrm{mg}$ every 2 days for 10 days. With these guideline recommendations in mind, it is not surprising that this study noted 12 unique regimens and a very wide range of doses (10-100 mg). The guidelines were unable to commit to a specific regimen, as there is significant heterogeneity in the literature. For CRSsP, the same 2011 CSO-HNS guidelines do not provide any dosing or duration suggestions but provide a statement supporting the use of a short course of OCSs in this condition [10]. The 2013 IFAR review by Poetker et al. provide an optional level $\mathrm{C}$ recommendation suggesting 40-60 mg for 10-14 days [11]. This heterogeneity and weakness in the literature for CRSsP, as well as CRSwP, may be the reason for wide variety of prescribing habits observed in this study. Regardless, some of the dosing regimens described by respondents fall outside of the broad spectrum of recommendations - either not providing patients with benefit if too low, or exposing them to unnecessary potential risk if too high.

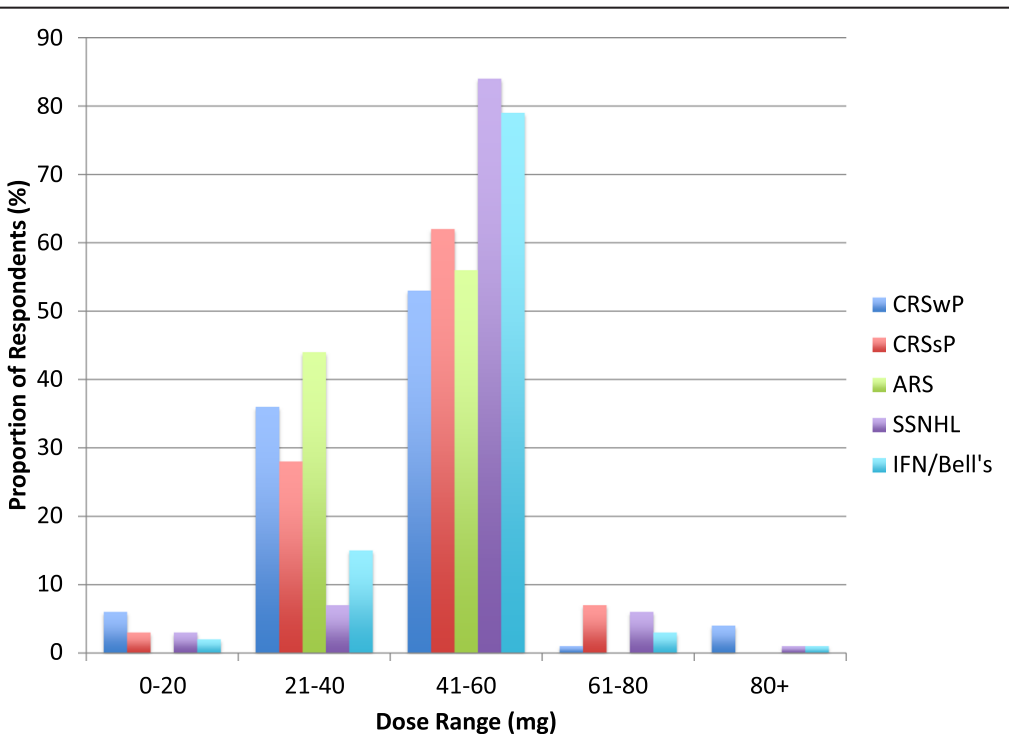

Fig. 3 Initial doses described by respondents for all conditions. Chronic Rhinosinusitis with Polyposis (Blue); Chronic Rhinosinusitis without Polyposis (Red); Acute Rhinosinusitis (Green); Sudden Sensori-neural Hearing Loss (Purple); Idiopathic Facial Nerve (Bell's) Palsy (Light Blue) 


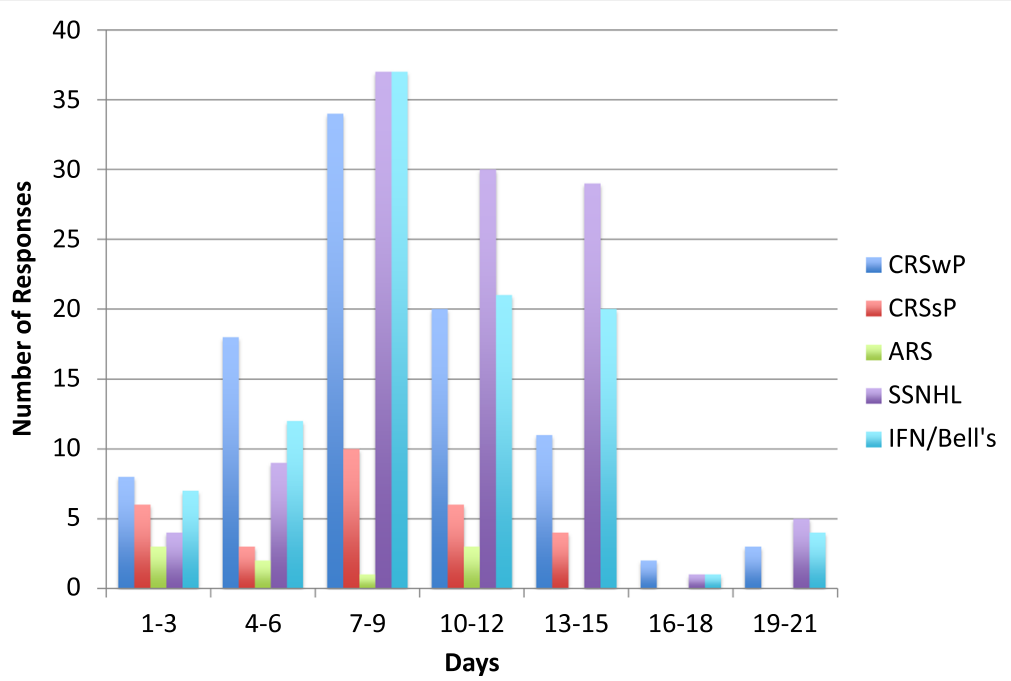

Fig. 4 Durations described by respondents for all conditions. Chronic Rhinosinusitis with Polyposis (Dark Blue); Chronic Rhinosinusitis without Polyposis (Red); Acute Rhinosinusitis (Green); Sudden Sensori-neural Hearing Loss (Purple); Idiopathic Facial Nerve (Bell's) Palsy (Light Blue)

The dosing and duration recommendations of corticosteroid for SSNHL and IFN palsy, on the other hand, is more granular. For SSNHL, the American Academy of Otolaryngology-Head and Neck Surgery clinical guidelines recommend the use of OCSs at $60 \mathrm{mg}$ for 10-14 days from level $B$ evidence [13]. In IFN palsy, the Canadian Medical Association Journal guidelines provide a strong recommendation for a five-day course of $60 \mathrm{mg}$ per day followed by a five day taper, reducing the previous day's dose by $10 \mathrm{mg}$ per day [14]. It is also suggested that a total dose of over $450 \mathrm{mg}$ is necessary to obtain optimal

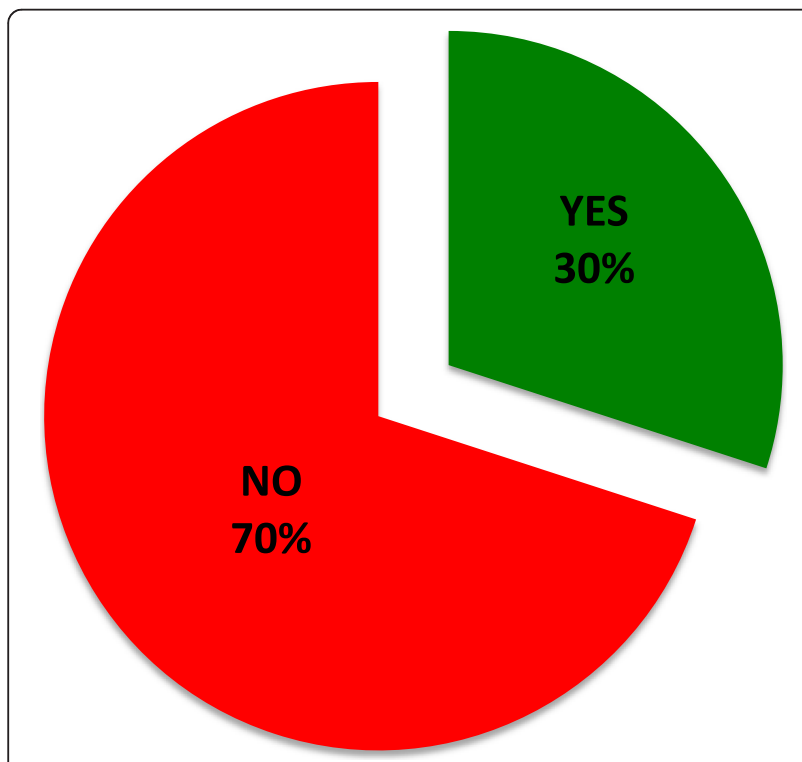

Fig 5 Percentage of respondents who have observed a complication with oral corticosteroids in their practice. Thirty percent of respondents answered "Yes" (Green) and $70 \%$ answered "No" (Red) benefit. Only $37 \%$ of respondents prescribed a dose over this total amount. The evidence supporting these recommendations is stronger than the literature for CRS, and should provide prescribers with greater guidance as to the ideal dose and duration of corticosteroid. The results of this study, in spite of stronger guideline recommendations, demonstrate that there still is a range in doses and duration for both conditions with only 80 and $67 \%$ of respondents using the recommended initial dose in SSNHL and IFN respectively. Prescribing below the guideline

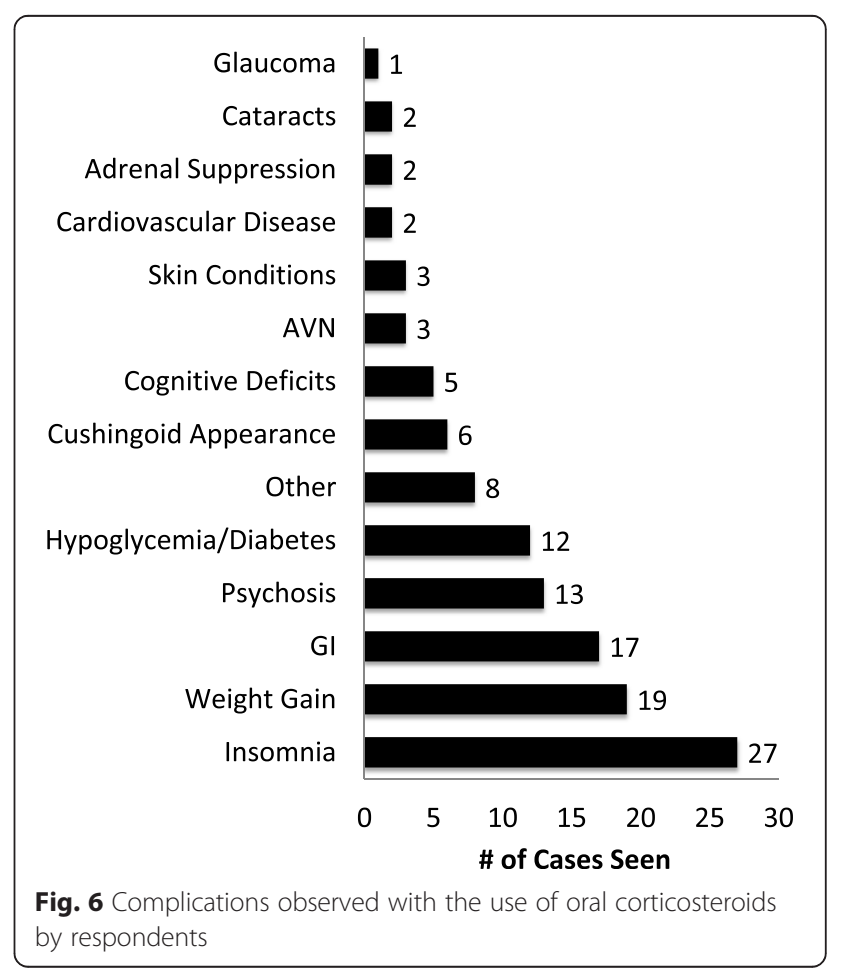


recommendations could potentially not provide patients with the intended benefit, while prescribing above could be exposing patients to unnecessary risk without any further additional benefit.

Evidence for corticosteroid use in acute rhinosinusitis is against routine use and not supported by current guideline recommendations $[10,15]$. In spite of this, $7 \%$ of respondents in this survey used OCSs in their treatment regimen, likely exposing patients to unnecessary risk without benefit.

Overall, this study was able to characterize current inpractice dosing regimens for five common conditions in which OCSs are used in OtoHNS. Further study needs to be performed to determine the optimal dose and duration for OCSs in CRS. However, there is significant heterogeneity in OCS prescribing habits irrespective of the strength of guideline recommendations regarding dose and duration. Perhaps greater emphasis is needed to encourage adherence to evidence-based practice to optimize medical therapy for patients.

Although this study aids in characterizing the dosing regimens of OCSs used in OtoHNS in Canada, it is self-reported. Hawthorne effect bias is likely present when respondents were completing this survey. Therefore, the results may be an under-estimation of the variability in the prescribing habits. This would further strengthen the conclusions made from this study. Additionally, it was difficult to parse out the reason for respondents to select "Yes" or "No" when replying to questions regarding the use of OCSs in each of the conditions mentioned in the survey. Some may have interpreted it as a question inquiring about the use of OCSs in their practice while others may have understood it as a question of whether they believed in prescribing OCSs for each specific condition. This would have potentially skewed the results of this question for each of the conditions. Further clarification of the question would have been optimal along with a third option of "Do not prescribe in my practice".

\section{Conclusion}

OCSs are widely used in OtoHNS. This study provides a glimpse into the in-practice prescription habits of Canadian Otolaryngologist-Head \& Neck surgeons for chronic rhinosinusitis with and without polyps, acute rhinosinusitis, sudden sensori-neural hearing loss, and idiopathic facial nerve palsy. As expected, there is a wide range of dosing regimens currently being used - some being within the recommended guidelines and others that are not. Further research to standardize prescribing habits in order to optimize patient outcomes and minimize potential risk from unnecessarily high doses of corticosteroid would be beneficial.

\section{Abbreviations}

ARS: acute rhinosinusitis; CRSsP: chronic rhinosinusitis without polyposis; CRSwP: chronic rhinosinusitis with polyposis; IFN: idiopathic facial nerve (Bell's) Palsy; OCS: oral corticosteroid; OtoHNS: Otolaryngology-Head and Neck Surgery; SSNHL: sudden sensori-neural hearing loss.

\section{Competing interests}

The authors declare that they have no other competing interests.

\section{Authors' contributions}

SA participated in the design of the study, carried out the survey, and drafted the manuscript. LJS conceived the study, participated in the study design, drafting and editing of the manuscript. BWR participated in the study design, drafting and editing of the manuscript. All authors read and approved the final manuscript.

\section{Acknowledgements}

This study was funded through the Schulich Research Opportunities Program (SROP) from the Schulich School of Medicine and Dentistry, Western University, London, Ontario. This funding body had no role in the design, collection, analysis, and interpretation of data; in the writing of the manuscript; and in the decision to submit the manuscript for publication.

\section{Confirmation of original material}

This material has never been published and is not currently under evaluation in any other peer-reviewed publication.

Received: 26 November 2015 Accepted: 8 February 2016

Published online: 29 February 2016

\section{References}

1. Overman RA, Yeh JY, Deal CL. Prevalence of oral glucocorticoid usage in the United States: a general population perspective. Arthritis Care Res (Hoboken). 2013;65(2):294-8.

2. Liu D, Ahmet A, Ward L, Krishnamoorthy P, Mandelcorn ED, Leigh R, et al. A practical guide to the monitoring and management of the complications of systemic corticosteroid therapy. Allergy Asthma Clin Immunol. 2013;9(1):30.

3. Manson SC, Brown RE, Cerulli A, Vidaurre CF. The cumulative burden of oral corticosteroid side effects and the economic implications of steroid use. Respir Med. 2009;103(7):975-94

4. Sylvester DB, Carr S, Nix P. Maximum medical therapy for chronic rhinosinusitis: a survey of otolaryngology consultants in the United Kingdom. Int Forum Allergy Rhinol. 2013;3(2):129-32.

5. Kaszuba SM, Stewart MG. Medical management and diagnosis of chronic rhinosinusitis: a survey of treatment patterns by United States otolaryngologists. Am J Rhinol. 2006;20:186-90.

6. Madana J, Morand GB, Barona-Lleo L, Black MJ, Mlynarek AM, Hier MP. A survey on pulmonary screening practices among Otolaryngology-Head \& Neck surgeons across Canada in the post treatment surveillance of head and neck squamous cell carcinoma. J Otolaryngol Head Neck Surg. 2015;44(1):5.

7. Williams BA, Trites JR, Taylor SM, Bullock MJ, Hart RD. Surgical management of primary hyperparathyroidism in Canada. J Otolaryngol Head Neck Surg. 2014;43(1):44.

8. Merdad M, Eskander A, De Almeida J, Freeman J, Rotstein L, Ezzat S, et al. Current Management of papillary thyroid microcarcinoma in Canada. J Otolaryngol Head Neck Surg. 2014;43:32

9. Brak M, Moore P, Taylor SM, Trites J, Murray S, Hart R. Expectantly waiting: a survey of thyroid surgery wait times among Canadian Otolaryngologists. J Otolaryngol Head Neck Surg. 2013:42:47.

10. Desrosiers M, Evans GA, Keith PK, Wright ED, Kaplan A, Bouchard J, et al. Canadian clinical practice guidelines for acute and chronic rhinosinusitis. J Otolaryngol Head Neck Surg. 2011;40 Suppl 2:S99-193.

11. Poetker DM, Jakubowski LA, Lal D, Hwang PH, Wright ED, Smith TL. Oral corticosteroids in the management of adult chronic rhinosinusitis with and without nasal polyps: an evidence-based review with recommendations. Int Forum Allergy Rhinol. 2013;3(2):104-20.

12. Kaplan A. Canadian guidelines for chronic rhinosinusitis: clinical summary. Can Fam Physician. 2013;59(12):1275-81.

13. Stachler RJ, Chandrasekhar SS, Archer SM, Rosenfeld RM, Schwartz SR, Barrs DM, et al. Clinical practice guideline: sudden hearing loss. Otolaryngol Head Neck Surg. 2012;146 Suppl 3:S1-S35. 
14. de Almeida JR, Guyatt GH, Sud S, Dorion J, Hill MD, Kolber MR. Management of Bell palsy: clinical practice guideline. CMAJ. 2014;186(12):917-22.

15. Venekamp RP, Thompson MJ, Hayward G, Heneghan CJ, Del Mar CB, Perera $\mathrm{R}$, et al. Systemic corticosteroids for acute sinusitis. Cochrane Database Syst Rev. 2014;25(3):CD008115.

Submit your next manuscript to BioMed Central and we will help you at every step:

- We accept pre-submission inquiries

- Our selector tool helps you to find the most relevant journal

- We provide round the clock customer support

- Convenient online submission

- Thorough peer review

- Inclusion in PubMed and all major indexing services

- Maximum visibility for your research

Submit your manuscript at www.biomedcentral.com/submit

Biomed Central 